\title{
ON DIFFERENCE EQUATION WITH GENERALIZED DILATION
}

PAVEL PLASCHINSKY

Received 22 July 2004; Revised 24 January 2005; Accepted 27 January 2005

We investigate the functional equation with generalized dilation in the special weighted functional spaces. We provide some sufficient conditions for the existence of the inversion operator in the same form and consider several examples.

Copyright (c) 2006 Pavel Plaschinsky. This is an open access article distributed under the Creative Commons Attribution License, which permits unrestricted use, distribution, and reproduction in any medium, provided the original work is properly cited.

\section{Introduction}

Consider the functional equation with generalized dilation:

$$
\sum_{n=1}^{\infty} a(n, x) n^{\nu \tau} f\left(n^{\tau} x\right)=g(x), \quad x \in(0 ; \infty), \tau, \nu \in \mathbb{R},
$$

where $a(n, x)$ is bounded almost everywhere on $(0 ; \infty)$ for arbitrary natural $n$, and the sequence $\left(a_{n}\right)$ of their $L_{\infty}$-norms belongs to $l_{1}$.

The equations of this type are used in many areas of physics [4, 5], for example, irradiation of black bodies. But in physics there were no rigorous proofs, rather it was the idea of using the method of the Dirichlet convolution inverse (we will call it here the discrete Mellin convolution).

One can find the expansive bibliography and history of the algebraic approach to the integral and difference equations with transformed argument in, for example, $[1-3,8]$.

The traditional use of the integral transforms in the case of constant coefficients does not work in $L_{p}$ Banach spaces, and we apply the method of the reciprocal sequences.

In [6], the functional operator $M_{a, \tau}$ on the left-hand side of (1.1) was completely investigated in the case of constant coefficients $a(n)$. In [7], it was shown that the operator $M_{a, \tau}$ is bounded in $L_{\gamma, p}$, that is, in the Banach space of functions $f(x)$ such that $f(x) x^{\nu-1 / p} \in L_{p}$ with the corresponding norm. In addition, sufficient conditions for the existence of the inversion operator of the same form as in (1.1) were found:

(1) $a(n, x)=\left(a_{1} * a_{2} * \cdots * a_{m}\right)_{\tau}(n, x)$;

(2) $\left\|a_{k}\right\|_{1}<\operatorname{essinf}\left|a_{k}(1, x)\right|+\operatorname{ess} \sup \left|a_{k}(1, x)\right|, k=1, \ldots, m$. 
2 On difference equation with dilation

In terms of the discrete Mellin convolution with $\tau$-degree dilation $\left(\mathrm{DMC}_{\tau}\right)$,

$$
(a * b)_{\tau}(n, x)=\sum_{k m=n} a(k, x) b\left(m, k^{\tau} x\right)
$$

these conditions imply the existence of the reciprocal sequence $\left(a^{-1}(n, x)\right)$ in $l_{1}$. The reciprocal sequence $\left(a^{-1}(n, x)\right)$ is defined almost everywhere on $(0 ; \infty)$ by equality

$$
\left(a * a^{-1}\right)_{\tau}(n, x)=\left(a^{-1} * a\right)_{\tau}(n, x)=e_{1}(n)= \begin{cases}1, & n=1, \\ 0, & n>1 .\end{cases}
$$

Under these conditions, the solution of (1.1) for arbitrary function $g(x)$ from $L_{\gamma, p}$ is of the form

$$
f(x)=\sum_{n=1}^{\infty} a^{-1}(n, x) n^{\nu \tau} g\left(n^{\tau} x\right), \quad x \in(0 ; \infty)
$$

since the inversion operator $M_{a, \tau}^{-1}$ is $M_{a^{-1}, \tau}$.

Furthermore, this form of solution is useful in calculations because of using concrete local values of functions, unlike using all values in integral transforms.

The goal of this paper is to find wider sufficient conditions in the case of arbitrary coefficients $a(n, x)$.

\section{Main theorem}

We denote

$$
\begin{aligned}
a_{\mathrm{inf}} & =\underset{(0 ; \infty)}{\operatorname{essinf}}|a(1, x)|, \quad a_{1}=\underset{(0 ; \infty)}{\operatorname{ess} \sup }|a(1, x)|, \\
a_{n}(\delta) & =\underset{(0 ; \delta)}{\operatorname{esssup}}\left|\frac{a(n, x)}{a(1, x)}\right|, \quad a_{n}(\Delta)=\underset{(\Delta ; \infty)}{\operatorname{esssup}}\left|\frac{a(n, x)}{a(1, x)}\right|, \\
a_{n}(0) & =\lim _{\delta \rightarrow 0} a_{n}(\delta), \quad a_{n}(\infty)=\lim _{\Delta \rightarrow \infty} a_{n}(\Delta), \\
a_{n} & =\underset{(0 ; \infty)}{\operatorname{esssup}}\left|\frac{a(n, x)}{a(1, x)}\right|, \quad n=2,3, \ldots .
\end{aligned}
$$

Theorem 2.1. If $a_{\mathrm{inf}}>0$ and

$$
\sum_{n=2}^{\infty} a_{n}(0)<1, \quad \sum_{n=2}^{\infty} a_{n}(\infty)<1,
$$

then there exists the reciprocal sequence $\left(a^{-1}(n, x)\right)$ with respect to the $D M C_{\tau}$ in $l_{1}$.

COROLlary 2.2. Under the conditions of the theorem, (1.1) has a unique solution and it can be expressed in the form (1.4).

Proof of the theorem. The condition $a_{\mathrm{inf}}>0$ is the necessary inversion condition for the sequence $a(n, x)$ [7]. Any sequence $(a(n, x)) \in l_{1}$ with this property can be introduced as 
the $\mathrm{DMC}_{\tau}$ of the sequences $(b(n, x))$ and $(c(n, x))$ belonging to $l_{1}$ :

$$
a(n, x)=(b * c)_{\tau}(n, x)
$$

where

$$
b(n, x)=\left\{\begin{array}{ll}
a(1, x), & n=1, \\
0, & n \geq 2,
\end{array} \quad c(n, x)= \begin{cases}1, & n=1 \\
\frac{a(n, x)}{a(1, x)}, & n \geq 2 .\end{cases}\right.
$$

The sequence $(b(n, x))$ is invertible in $l_{1}$ and

$$
b(n, x)= \begin{cases}\frac{1}{a(1, x)}, & n=1 \\ 0, & n \geq 2\end{cases}
$$

If the sequence $(c(n, x))$ is also invertible in $l_{1}$, then the sequence $(a(n, x))$ is invertible and [7]

$$
a^{-1}(n, x)=\left(c^{-1} * b^{-1}\right)_{\tau}(n, x)
$$

The proof of the existence of $\left(c^{-1}(n, x)\right)$ in $l_{1}$ will be divided into several parts.

(1) There are two positive numbers $\delta$ and $\Delta$ such that

$$
\sum_{n=2}^{\infty} a_{n}(\delta)<1, \quad \sum_{n=2}^{\infty} a_{n}(\Delta)<1
$$

Indeed, as $(c(n, x)) \in l_{1}$, for arbitrary $\varepsilon>0$ there is a natural $N$ such that

$$
\sum_{n=N+1}^{\infty} \underset{(0 ; \infty)}{\operatorname{essup}}\left|\frac{a(n, x)}{a(1, x)}\right|<\varepsilon
$$

Moreover, for any $n=2, \ldots, N$, there are two positive numbers $\delta_{n}$ and $\Delta_{n}$ such that

$$
\underset{\left(0 ; \delta_{n}\right)}{\operatorname{esssup}}\left|\frac{a(n, x)}{a(1, x)}\right| \leq a_{n}(0)+\frac{\varepsilon}{N}, \quad \underset{\left(\Delta_{n} ; \infty\right)}{\operatorname{esssup}}\left|\frac{a(n, x)}{a(1, x)}\right| \leq a_{n}(\infty)+\frac{\varepsilon}{N} .
$$

Denoting the $\min \left\{\delta_{n}\right\}$ by $\delta$ and the $\max \left\{\Delta_{n}\right\}$ by $\Delta$, we obtain the inequalities

$$
\begin{aligned}
& \sum_{n=2}^{\infty} a_{n}(\delta)<\sum_{n=2}^{N} a_{n}(0)+2 \varepsilon<\sum_{n=2}^{\infty} a_{n}(0)+2 \varepsilon<1, \\
& \sum_{n=2}^{\infty} a_{n}(\Delta)<\sum_{n=2}^{N} a_{n}(\infty)+2 \varepsilon<\sum_{n=2}^{\infty} a_{n}(\infty)+2 \varepsilon<1,
\end{aligned}
$$

since $\varepsilon$ may be taken arbitrarily small. 
4 On difference equation with dilation

(2) There is an explicit inversion formula [7]:

$$
c^{-1}(n, x)=\sum_{\beta \in A_{n}}(-1)^{|\beta|} \sum_{i(\beta)} \prod_{k=1}^{|\beta|} c\left(i_{k}, i_{1}^{\tau} i_{2}^{\tau} \cdots i_{k-1}^{\tau} x\right)
$$

where

$$
A_{n}=\left\{\beta=\left(\beta_{2}, \beta_{3}, \ldots, \beta_{n}\right), \beta_{m}=0,1,2, \ldots \mid \prod_{m=2}^{n} m^{\beta_{m}}=n, n \geq 2\right\}
$$

$i(\beta)=$ the set of permutations of the natural numbers corresponding to $\beta_{m} \neq 0$ (the number $m$ is taken $\beta_{m}$ times),

$$
|\beta|=\sum_{m=2}^{n} \beta_{m}
$$

Thus we need to show that the sum

$$
\left\|c^{-1}\right\| \leq \sum_{n=1}^{\infty} \sum_{\beta \in A_{n}} \sum_{i(\beta)} \prod_{k=1}^{|\beta|} \underset{(0 ; \infty)}{\operatorname{esssup}}\left|\frac{a\left(i_{k}, i_{1}^{\tau} i_{2}^{\tau} \cdots i_{k-1}^{\tau} x\right)}{a\left(1, i_{1}^{\tau} i_{2}^{\tau} \cdots i_{k-1}^{\tau} x\right)}\right|
$$

is finite. Without loss of generality, we may assume that $\tau>0$.

(3) On the interval $(\Delta ; \infty)$, we obtain as in [7]

$$
\begin{aligned}
\sum_{n=1}^{\infty} \underset{(\Delta ; \infty)}{\operatorname{esssup}}\left|c^{-1}(n, x)\right| & \leq \sum_{n=1}^{\infty} \sum_{\beta \in A_{n}} \frac{|\beta| !}{\beta !} a^{\beta}(\Delta)=\sum_{s=0}^{\infty} \sum_{|\beta|=s} \frac{s !}{\beta !} a^{\beta}(\Delta) \\
& =\sum_{s=0}^{\infty}\left(\sum_{n=2}^{\infty} a_{n}(\Delta)\right)^{s}=\left(1-\sum_{n=2}^{\infty} a_{n}(\Delta)\right)^{-1}<\infty .
\end{aligned}
$$

Here

$$
\beta !=\prod_{m=2}^{n} \beta_{m} !, \quad a^{\beta}(\Delta)=\prod_{m=2}^{n} a_{m}^{\beta_{m}}(\Delta)
$$

and the final equality is obtained as the sum of decreasing infinite geometric progression in view of (2.7).

(4) We investigate the sum (2.14) on the interval $(\delta ; \Delta)$.

Without the loss of generality, we may assume that $\Delta=\delta 2^{L \tau}$, where $L \in \mathbb{N}$. In every product from (2.14), we will take the factors with condition $i_{1} \cdots i_{k-1} \leq 2^{L}$. At least one such factor is taken, because the first one depends only on $x$.

We say that a shift is a boundary shift with respect to the $2^{L}$ if $i_{1} \cdots i_{k-1} \leq 2^{L}$, but $i_{1} \cdots i_{k-1} 2>2^{L}$. We say that a shift is an inner shift if $i_{1} \cdots i_{k-1} 2 \leq 2^{L}$. 
We first estimate the sum from (2.14) with inner shifts in the product. The number of multiples is at most $L$, and similarly to the previous case, we obtain the number

$$
\left(1-\left(\sum_{n=2}^{\infty} a_{n}\right)^{L+1}\right) \times\left(1-\sum_{n=2}^{\infty} a_{n}\right)^{-1}
$$

as an upper bound of the sum.

We group the rest of the sum (2.14) according to the common beginning of the products, bounded by the factor with a boundary shift. Taking the common beginning out of each group, we thus obtain the same sum within the boundary shift $\left(i_{1} \cdots i_{k}\right)^{\tau}$.

Indeed, the sum (2.14) consists of various finite products of the norms of the elements $c(n, x)$ with special type shifts. If we take out a part of the sum with the same beginning, then it also consists of various finite remainders of products within the considered shift, which is the same sum as in (2.14).

In view of $\left(i_{1} \cdots i_{k}\right)^{\tau} \delta \geq 2^{L \tau} \delta=\Delta$, this sum can be bounded by the number

$$
\left(1-\sum_{n=2}^{\infty} a_{n}(\Delta)\right)^{-1}
$$

As the sum of the common beginnings consists of at most $L+1$ multiples in a summand, the upper bound is

$$
\left(1-\left(\sum_{n=2}^{\infty} a_{n}\right)^{L+2}\right) \times\left(1-\sum_{n=2}^{\infty} a_{n}\right)^{-1} .
$$

Combining all the results thus obtained on the interval $(\delta ; \Delta)$, we have

$$
\begin{aligned}
& \sum_{n=1}^{\infty} \underset{(\delta ; \Delta)}{\operatorname{esssup}}\left|c^{-1}(n, x)\right| \\
& \leq\left(1-\sum_{n=2}^{\infty} a_{n}\right)^{-1} \times\left[1-\left(\sum_{n=2}^{\infty} a_{n}\right)^{L+1}+\left(1-\left(\sum_{n=2}^{\infty} a_{n}\right)^{L+2}\right)\left(1-\sum_{n=2}^{\infty} a_{n}(\Delta)\right)^{-1}\right]<\infty
\end{aligned}
$$

(5) We finally investigate the norm on each interval $\left(2^{-(K+1) \tau} \delta ; 2^{-K \tau} \delta\right)$, where $K$ is a nonnegative integer.

As for the previous interval, we first consider the sum with inner shifts with respect to $2^{K}$. By analogy, the number

$$
\left(1-\left(\sum_{n=2}^{\infty} a_{n}(\delta)\right)^{K+1}\right) \times\left(1-\sum_{n=2}^{\infty} a_{n}(\delta)\right)^{-1}
$$

is an upper bound of the sum.

The rest of the sum (2.14), for the same reason, is bounded by the number

$$
\frac{1-\left(\sum_{n=2}^{\infty} a_{n}(\delta)\right)^{K+2}}{1-\sum_{n=2}^{\infty} a_{n}(\delta)} \cdot \frac{1-\left(\sum_{n=2}^{\infty} a_{n}\right)^{L+2}}{1-\sum_{n=2}^{\infty} a_{n}} \cdot \frac{1}{1-\sum_{n=2}^{\infty} a_{n}(\Delta)}
$$


Here the first fraction corresponds to the sum of products bounded by the multiples with boundary shifts with respect to $2^{K}$ (see (2.7)); the second one corresponds to the sum with shifts in the interval $\left(2^{K} ; 2^{K+L+1}\right)$; the last one corresponds to the common sum within the shift as in the previous case.

Since for arbitrary $K$,

$$
1-\left(\sum_{n=2}^{\infty} a_{n}(\delta)\right)^{K+2} \leq 1
$$

the sum $(2.14)$ is bounded on the interval $(0 ; \delta)$. This completes the proof of the theorem.

\section{Some applications}

Example 3.1. The equation

$$
f(x)-a(x) 2^{1 / p} f(2 x)=g(x), \quad x \in(0 ; \infty),
$$

where $g(x) \in L_{p}$, if

$$
\lim _{\mathcal{\varepsilon} \rightarrow+0} \operatorname{esssup}|a(x)|<1, \quad \lim _{E \rightarrow \infty} \operatorname{esssup} \underset{(E ; \infty)}{\operatorname{exs}}|a(x)|<1
$$

has a unique solution in $L_{p}$ :

$$
f(x)=\sum_{n=0}^{\infty}\left(\prod_{k=0}^{n-1} a\left(2^{k} x\right)\right) 2^{n / p} g\left(2^{n} x\right)
$$

in view of Corollary 2.2 and (1.4) (here $\tau=1, \nu=1 / p$ ).

Example 3.2. We investigate the equation

$$
f(x)-x e^{1-x} 2^{1 / p} f(2 x)=g(x), \quad x \in(0 ; \infty),
$$

when $g(x) \in L_{p}$.

It is easy to see that $\max \left|-x e^{1-x}\right|=1$, that is, the sufficient condition from [7] fails. But the formula (3.3) allows us to find the solution in $L_{p}$ as

$$
f(x)=e^{x} \sum_{n=0}^{\infty}(x e)^{n}(\sqrt{2})^{n(n-1)} e^{-2^{n} x} 2^{n / p} g\left(2^{n} x\right),
$$

because the functional sequence $(a(n, x))=\left(1,-x e^{1-x}, 0, \ldots\right)$ is invertible in $l_{1}$ and

$$
a^{-1}\left(2^{n}, x\right)=\prod_{k=0}^{n-1} a\left(2,2^{k} x\right)=\prod_{k=0}^{n-1} 2^{k} x e^{1-2^{k} x}=(x e)^{n}(\sqrt{2})^{n(n-1)} e^{-\left(2^{n}-1\right) x}
$$

for every nonnegative integer $n$. 
Example 3.3. To generalize Example 3.1, the equation

$$
f(x)-a(x) m^{1 / p} f(m x)=g(x), \quad x \in(0 ; \infty), m=2,3, \ldots,
$$

with the same assumptions has a unique solution in $L_{p}$ :

$$
f(x)=\sum_{n=0}^{\infty}\left(\prod_{k=0}^{n-1} a\left(m^{k} x\right)\right) m^{n / p} g\left(m^{n} x\right) .
$$

Example 3.4. The equation

$$
f(x)-2 a(x) 2^{1 / p} f(2 x)+a(x) a(2 x) 4^{1 / p} f(4 x)=g(x), \quad x \in(0 ; \infty),
$$

has a unique solution in $L_{p}$ for arbitrary $g(x) \in L_{p}$ if the conditions of Example 3.1 are satisfied.

Indeed, Theorem 2.1 requires

$$
\begin{gathered}
2 a(0)+a^{2}(0)<1, \quad \text { where } a(0)=\lim _{\varepsilon \rightarrow+0} \underset{(0 ; \varepsilon)}{\operatorname{esssup}}|a(x)|, \\
2 a(\infty)+a^{2}(\infty)<1, \quad \text { where } a(\infty)=\lim _{E \rightarrow \infty} \underset{(E ; \infty)}{\operatorname{ess} \sup }|a(x)| .
\end{gathered}
$$

This implies that $a(0)<\sqrt{2}-1$ and $a(\infty)<\sqrt{2}-1$.

But the sequence $(b(n, x))=(1,-2 a(x), 0, a(x) a(2 x), 0, \ldots)$ can be rewritten in the form of $\mathrm{DMC}_{1}: b(n, x)=(a * a)_{1}(n, x)$, where $(a(n, x))=(1,-a(x), 0, \ldots)$.

Applying Theorem 2.1 to the functional sequence $(a(n, x))$, we obtain the same conditions as in Example 3.1.

The solution of the equation depends on the reciprocal sequence $b^{-1}$,

$$
\begin{aligned}
b^{-1}\left(2^{n}, x\right) & =\left(a^{-1} * a^{-1}\right)_{1}\left(2^{n}, x\right) \\
& =\sum_{k+m=n} a^{-1}\left(2^{k}, x\right) a^{-1}\left(2^{m}, 2^{k} x\right)=\sum_{k+m=n} \prod_{i=0}^{k-1} a\left(2^{i} x\right) \prod_{j=0}^{m-1} a\left(2^{j+k} x\right),
\end{aligned}
$$

and is of the form

$$
f(x)=\sum_{n=0}^{\infty} b^{-1}\left(2^{n}, x\right) 2^{n / p} g\left(2^{n} x\right) .
$$

\section{Acknowledgment}

I would like to thank A. B. Antonevich for the attention to the paper. 
8 On difference equation with dilation

\section{References}

[1] A. Antonevich, M. Belousov, and A. Lebedev, Functional Differential Equations. II. C*Applications. Part 1. Equations with Continuous Coefficients, Pitman Monographs and Surveys in Pure and Applied Mathematics, vol. 94, Longman, Harlow, 1998.

[2] _ Functional Differential Equations. II. $C^{*}$-Applications. Part 2. Equations with Discontinuous Coefficients and Boundary Value Problems, Pitman Monographs and Surveys in Pure and Applied Mathematics, vol. 95, Longman, Harlow, 1998.

[3] A. Antonevich and A. Lebedev, Functional-Differential Equations. I. $C^{*}$-Theory, Pitman Monographs and Surveys in Pure and Applied Mathematics, vol. 70, Longman Scientific \& Technical, Harlow, 1994.

[4] N. X. Chen, Modified Möbius inverse formula and its applications in physics, Physical Review Letters 64 (1990), no. 11, 1193-1195.

[5] R. Estrada, Dirichlet convolution inverses and solution of integral equations, Journal of Integral Equations and Applications 7 (1995), no. 2, 159-166.

[6] P. V. Plaschinsky, Discrete Mellin convolution with dilation and its applications, Mathematical Modelling and Analysis 3 (1998), 160-167.

[7] _ One functional operator inversion formula, Mathematical Modelling and Analysis 6 (2001), no. 1, 138-146.

[8] D. Przeworska-Rolewicz, Equations with Transformed Argument. An Algebraic Approach, Elsevier Scientific Publishing, Amsterdam; PWN—Polish Scientific, Warsaw, 1973.

Pavel Plaschinsky: Faculty of Mathematics and Mechanics, Belarusian State University,

4 F. Skaryny Avenue, Minsk, Belarus 220050

E-mail address: plaschinsky@bsu.by 